VOL. 45 (1992) [513-520]

\title{
GROUPS WITH A QUOTIENT THAT CONTAINS THE ORIGINAL GROUP AS A DIRECT FACTOR
}

\author{
Ron Hirshon and David Meier
}

\begin{abstract}
We prove that given a finitely generated group $G$ with a homomorphism of $G$ onto $G \times H, H$ non-trivial, or a finitely generated group $G$ with a homomorphism of $G$ onto $G \times G$, we can always find normal subgroups $N \neq G$ such that $G / N \cong$ $G / N \times H$ or $G / N \cong G / N \times G / N$ respectively. We also show that given a finitely presented non-Hopfian group $U$ and a homomorphism $\varphi$ of $U$ onto $U$, which is not an isomorphism, we can always find a finitely presented group $H \supseteq U$ and a finitely generated free group $F$ such that $\varphi$ induces a homomorphism of $U * F$ onto $(U * F) \times H$. Together with the results above this allows the construction of many examples of finitely generated groups $G$ with $G \cong G \times H$ where $H$ is finitely presented. A finitely presented group $G$ with a homomorphism of $G$ onto $G \times G$ was first constructed by Baumslag and Miller. We use a slight generalisation of their method to obtain more examples of such groups.
\end{abstract}

\section{INTRODUCTION}

Finitely generated groups $G$ with $G \cong G \times H, H \neq 1$, or $G \cong G \times G$ were first constructed by Tyrer Jones [4], but it is still an open question whether there exist non-trivial finitely presented groups $G$ with these properties. More insight into the structure and a new construction of finitely generated groups with the above properties are therefore still useful. The starting point of our investigations was the construction of a finitely presented group $G$ with a homomorphism of $G$ onto $G \times G$ by Baumslag and Miller [1].

Our paper consists of four sections. The main results are the following theorems which are proved in Sections 3 and 5 respectively. Theorem 2: A group $G$ with a homomorphism of $G$ onto $G \times H$ always contains a normal subgroup $N$ such that $G / N \cong G / N \times H$. Theorem 4: A non-trivial group $G$ with a homomorphism from $G$ onto $G \times G$ always contains a normal subgroup $N \neq G$ such that $G / N \cong G / N \times G / N$.

Since in Sections 3 and 5 we need finitely presented groups $G$ with homomorphisms of $G$ onto $G \times H$ or of $G$ onto $G \times G$, we study the construction of such groups in

\footnotetext{
Received 28 June 1991
}

We would like to thank the referee for his constructive comments to an earlier version of our paper which have improved its presentation.

Copyright Clearance Centre, Inc. Serial-fee code: 0004-9729/92 \$A2.00+0.00. 
Sections 2 and 4. In Section 2 we use ideas contained in [3] to give a fairly general construction for groups $G$ with a homomorphism of $G$ onto $G \times H$. More precisely, we show that given a finitely presented non-Hopfian group $U$ and a homomorphism $\varphi$ of $U$ onto $U$, which is not an isomorphism, we can always find a finitely presented group $\boldsymbol{H} \supseteq U$ and a finitely generated free group $F$ such that $\varphi$ induces a homomorphism $\vartheta$ of $U * F$ onto $(U * F) \times H$. We use this to give an example of a one-relator group $G$ with a homomorphism of $G$ onto $G \times H, H \neq 1$. Together with Theorem 2 this allows the construction of many examples of finitely generated groups $G$ with $G \cong G \times H$ where $H$ is finitely presented. The construction of groups $G$ with a homomorphism of $G$ onto $G \times G$ given in Section 4 is just a slight generalisation, again based on ideas in [3], of the Baumslag-Miller group [1]. We start with a finitely generated abelian group $A$ and a one-one homomorphism $\mu$ of $A$ to $A$ satisfying some additional conditions and construct a finitely presented group $G \supseteq A$ and a homomorphism $\vartheta$ from $G$ onto $G \times G$ induced by $\mu$.

\section{FintTELy PRESENTED GROUPS $G$ WITH A HOMOMORPHISM FROM $G$ ONTO $G \times H$}

The following lemma is needed in the proof of Theorem 1.

LEMMA 1. Let $U$ be finitely presented and $1 \neq x \in U$. Then there exists a finitely presented group $H \supseteq U$, such that $x$ generates $H$ as a normal subgroup.

A proof can, for example, be modelled after the proof of Theorem 3.5 of [2], p.190.

Theorem 1. Let $U$ be finitely presented and non-Hopfian and let $\varphi$ be a homomorphism of $U$ onto $U$ with non-trivial kernel. Then there exists a finitely presented group $H \supseteq U$ and a finitely presented group $V$ such that $\varphi$ induces a homomorphism $\vartheta$ of $V * U$ onto $(V * U) \times H$.

Proof: Let $1 \neq x$ be an element in the kernel of $\varphi$. Form the group $H$ as in Lemma 1 and let $\mu: U \rightarrow H$ be the embedding. Let $V$ be any finitely presented group for which there exists a homomorphism $\rho$ of $V$ onto $H$. The map $\vartheta$ of $V * U$ onto $(V * U) \times H$ is given by

$$
\begin{aligned}
& \vartheta: v \rightarrow(v, v \rho) \text { for } v \in V \text { of } V * U \\
& \vartheta: u \rightarrow(u \varphi, u \mu) \text { for } u \in U \text { of } V * U .
\end{aligned}
$$

In $(u \varphi, u \mu)$ the left-hand side, $u \varphi$, is in $U$ of $V * U$. Because $V * U$ is a free product and $\varphi$ and $\rho$ are homomorphisms, $\vartheta$ extends to a homomorphism. Note, $x \vartheta=(1, x \mu)$. The map $\rho$ is onto. $(V * U) \vartheta$ contains therefore an element $(g, h)$ for all $h \in H$. Since $x \mu$ generates $H$ as a normal subgroup, $(V * U) \vartheta$ contains $(1, h)$ for all $h \in H$ and 
therefore $(1, H)$. Therefore $(V * U) \vartheta$ contains $(v, 1)$ for all $v \in V$ and $(u \varphi, 1)$ for all $u \in U$. Because $\varphi$ is onto this implies that also $(V * U, 1)$ of $(V * U) \times H$ is in $(V * U) \vartheta$. Together we get that $\vartheta$ is onto and the proof is complete.

REMARK. There are two natural choices for. $V$ :

(i) $V=H$ and $\rho$ the identity map.

(ii) $V$ free. More exactly if $h_{1}, \ldots, h_{n}$ is a generating set of $B$ then we may take $V=\left\langle f_{1}, \ldots, f_{n}\right\rangle$, the free group freely generated by $f_{1}, \ldots, f_{n}$ and $\rho$ the homomorphism given by $f_{i} \rho=h_{i}$ for $i=1, \ldots, n$. This gives the following corollary:

COROLLARY 1. Let $U$ be finitely presented and non-Hopfian. Then there exists a finitely generated free group $F$ and a finitely presented group $H$ containing $U$ such that there is a homomorphism $\vartheta$ of $F * U$ onto $(F * U) \times H$.

EXAMPLE 1: Let $U=\left\langle t, a ; t^{-1} a^{2} t=a^{3}\right\rangle$ be the well known non-Hopfian group of Baumslag and Solitar. See [2], p.197, where it is also shown that $1 \neq x=\left[t^{-1} a t, a\right]$ is in the kernel of $\varphi$ given by $t \varphi=t$ and $a \varphi=a^{2}$. The group

$$
H=\left\langle t, a, s, b ; t^{-1} a^{2} t=a^{3}, s^{-1} b^{2} s=b^{3}, s=\left[t^{-1} a t, a\right], t=\left[s^{-1} b s, b\right]\right\rangle,
$$

which contains $U$ and has the property that $x$ generates $H$ as a normal subgroup, was constructed in [3]. Let $G_{1}$ be the free product of $H$ and $U$ :

$$
\begin{array}{r}
G_{1}=\left\langle t, a, s, b, r, c ; t^{-1} a^{2} t=a^{3}, s^{-1} b^{2} s=b^{3}, s=\left[t^{-1} a t, a\right],\right. \\
\left.t=\left[s^{-1} b s, b\right], r^{-1} c^{2} r=c^{3}\right\rangle
\end{array}
$$

By Theorem 1, the homomorphism $\vartheta$ of $G_{1}$ to $G_{1} \times H$ given by

$$
\begin{aligned}
& \vartheta: t \rightarrow(t, t), \vartheta: a \rightarrow(a, a), \vartheta: s \rightarrow(s, s), \vartheta: b \rightarrow(b, b), \\
& \vartheta: r \rightarrow(r, t), \vartheta: c \rightarrow\left(c^{2}, a\right)
\end{aligned}
$$

is onto.

EXAMPLE 2: Let $U$ and $H$ be the groups of Example 1. The relation $s=$ $\left[t^{-1} a t, a\right]$ of $H$ can be used to eliminate the generator $s$, so that $H$ has the following presentation on three generators and three relations:

$$
H=\left\langle t, a, b ; t^{-1} a^{2} t=a^{3},\left[a, t^{-1} a t\right] b^{2}\left[t^{-1} a t, a\right]=b^{3}, t=\left[\left[a, t^{-1} a t\right] b\left[t^{-1} a t, a\right], b\right]\right\rangle .
$$

Let $G_{2}$ be the one-relator group

$$
G_{2}=\left\langle r, c, f_{t}, f_{a}, f_{b} ; r^{-1} c^{2} r=c^{3}\right\rangle .
$$

Then by Corollary 1 , the map $\vartheta$ of $G_{2}$ to $G_{2} \times H$ given by

$$
\begin{aligned}
& \vartheta: f_{t} \rightarrow\left(f_{t}, t\right), \vartheta: f_{a} \rightarrow\left(f_{a}, a\right), \vartheta: f_{b} \rightarrow\left(f_{b}, b\right), \\
& \vartheta: r \rightarrow(r, t), \vartheta: c \rightarrow\left(c^{2}, a\right)
\end{aligned}
$$

is onto. 


\section{Finitely Generated GRoups $G$ With $G \cong G \times H$, $H$ FINITELY PRESENTED}

Theorem 2. Let $\vartheta$ be a homomorphism of $A$ onto $A \times B$. Then $A$ contains a normal subgroup $K$ such that $A / K \cong A / K \times B$.

ProOF: Let $K$ be a maximal normal subgroup such that $K \vartheta$ is contained in $(K, 1)$. Then $\vartheta$ induces a map $\eta$ of $A / K$ onto $A / K \times B$. Let $L=\operatorname{ker} \eta$ and let $N$ be its preimage under the quotient map $A \rightarrow A / K$. Then $N$ contains $K$ and has the property that $N \vartheta$ is contained in $(N, 1)$. By the maximality of $K$, we get that $K=N$ and therefore that $\eta$ is an isomorphism and the proof is complete.

We now show that a maximal normal subgroup $K$ of $A$ with the property that $K \vartheta$ is contained in $(K, 1)$ is unique.

THE UPPER KERNEL OF A HOMOMORPHISM $\vartheta$ OF $A$ ONTO $A \times B$. Let $A_{1}=(A, 1) \vartheta^{-1}$ and for $i>0$, let $A_{i+1}=\left(A_{i}, 1\right) \vartheta^{-1}$. The $A_{i}$ form a descending chain of normal subgroups. The intersection of the $A_{i}$ contains the kernel of $\vartheta$. We call it the upper kernel of $\vartheta$ and write uker $(\vartheta)$ for it and claim that it has the property $A /$ uker $(\vartheta) \cong$ $A /$ uker $(\vartheta) \times B$. Since $\vartheta$ maps all elements of uker $(\vartheta)$ to (uker $(\vartheta), 1), \vartheta$ induces a map $\vartheta^{\prime}$ from $A / \operatorname{uker}(\vartheta)$ to $A / \operatorname{uker}(\vartheta) \times B$, and since $a \vartheta \in(\operatorname{uker}(\vartheta), 1)$ implies $a \in \operatorname{uker}(\vartheta), \vartheta^{\prime}$ is an isomorphism.

REMARK. If $a \vartheta \in(A, 1)$, we can consider $a \vartheta$ as an element of $A$ and apply $\vartheta$ again to get $a \vartheta^{2}$. If $a \vartheta^{2}$ is again in $(A, 1)$ we can continue in the same way to get $a \vartheta^{3}$ and so on. The upper kernel uker $(\vartheta)$ consists now precisely of those elements $a \in A$ with $a \vartheta^{n}$ in $(A, 1)$ for all $n$.

Proposition 1. Let $\vartheta$ be a homomorphism of $A$ onto $A \times B$. If $K$ is a maximal normal subgroup of $A$ such that $K \vartheta$ is contained in $(K, 1)$, then $K=$ uker $(\vartheta)$.

Proof: Since $K \vartheta$ is contained in $(K, 1), A_{1}=(A, 1) \vartheta^{-1} \supseteq K$. Assume now that $A_{i} \supseteq K$. Then $K \supseteq K \vartheta$ implies $A_{i} \supseteq K \vartheta$ and $A_{i+1}=\left(A_{i}, 1\right) \vartheta^{-1} \supseteq K$. By induction we get $A_{i} \supseteq K$ for all $i$ and therefore uker $(\vartheta) \supseteq K$. By maximality of $K$ this implies $K=\operatorname{uker}(\vartheta)$.

ThE LOWER KERNEL OF A HOMOMORPhisM $\vartheta$ OF $A$ onTo $A \times B$. The upper kernel is a method of finding the largest normal subgroup $K$ with $K \vartheta$ in $(K, 1)$ and $A / K \cong$ $A / K \times B$ as an intersection of a chain of subgroups. The smallest normal subgroup with $K \vartheta$ in $(K, 1)$ and $A / K \cong A / K \times B$ can be found as follows. Let $K_{1}=\operatorname{ker}(\vartheta)$. Then $\vartheta$ induces a map $\vartheta_{1}$ of $A / K_{1}$ onto $A / K_{1} \times B$. Let $K_{2}$ be the preimage of $\operatorname{ker}\left(\vartheta_{1}\right)$ under the quotient map $A$ to $A / K_{1}$. Inductively if $\vartheta_{i}$ maps $A / K_{i}$ onto $A / K_{i} \times B$ we define $K_{i+1}$ to be the preimage of $\operatorname{ker}\left(\vartheta_{i}\right)$ under the quotient map $A$ to $A / K_{i}$. The $K_{i}$ form an ascending chain of subgroups. Let $K=\cup K_{i}$. We call $K$ the lower kernel 
of $\vartheta$ and write lker $(\vartheta)$ for it. $\vartheta$ induces a map $\vartheta^{\prime}$ from $A / K$ onto $A / K \times B$ which is an isomorphism.

Proposition 2. Let $\vartheta$ be a homomorphism of $A$ onto $A \times B$. If $N$ is a normal subgroup of $A$ such that $N \vartheta$ is contained in $(N, 1)$ and $\vartheta$ induces an isomorphism of $A / N$ to $A / N \times B$, then $N \supseteq \operatorname{lker}(\vartheta)$.

Proof: Obviously, $N \supseteq K_{1}=\operatorname{ker}(\vartheta)$. Assume now that $N \supseteq K_{i}$. Then $K_{i+1} N / N$ is in the kernel of the homomorphism $A / N$ to $A / N \times B$ induced by $\vartheta$. Since this map is an isomorphism, $N \supseteq K_{i+1}$. By induction we get $N \supseteq K_{i}$ for all $i$, therefore $N \supseteq \operatorname{lker}(\vartheta)$.

REMARK. In general $\operatorname{lker}(\vartheta) \neq u$ uker $(\vartheta)$. If $G \cong G \times B$ and $\varphi$ is an isomorphism of $G$ to $G \times B$ and $H$ is any group then the map $\vartheta$ of $H \times G$ to $(H \times G) \times B$ given by $(h, g) \vartheta=(h, g \varphi)$ is an isomorphism. In this case we get $\operatorname{lker}(\vartheta)=1$ and uker $(\vartheta) \supseteq H$.

Corollary 2. Let $U$ be a finitely presented non-Hopfian group. Form the finitely presented group $B$ as in Lemma 1. Then there exists a finitely generated group $G$ such that $G \cong G \times H$.

Proof: By Theorem 1, there exists a homomorphism $\vartheta$ of $H * U$ onto $H * U \times H$. By Theorem 2, the group $G=H * U /$ uker $(\vartheta)$ has the property $G \cong G \times H$.

EXAMPLE 3: Let $G_{2}$ and $H$ be as in Example 2. From the above, there exists a quotient $G_{3}$ of $G_{2}$ such that $G_{3} \cong G_{3} \times H$.

\section{Finitely PRESENTED GROUPS $G$ WITH A HOMOMORPHISM FROM $G$ ONTO $G \times G$}

Let $A=\left\langle a_{1}, \ldots, a_{n}\right\rangle$ be a finitely generated abelian group and let $\mu, \nu$ be two oneone homorphisms from $A$ to $A$ which are not onto, such that $\mu \nu=\nu \mu$. It was shown in [3] that the HNN extension $U=\left\langle t, A ; t^{-1} a_{i} \mu t=a_{i} \nu, i=1, \ldots, n\right\rangle$ is non-Hopfian if $A=\langle A \mu, A \nu\rangle$. Theorem 3 generalises the construction of the group of Theorem $\mathrm{C}$ of Baumslag and Miller [1].

TheOREM 3. Let $A, \mu, \nu$ be as above. If the elements $a_{i} \mu\left(a_{i} \nu\right)^{-1}, i=1, \ldots, n$ generate $A$, then there exists a finitely presented group $G$ containing $A$ with a homomorphism $\vartheta$ of $G$ onto $G \times G$ induced by $\mu$.

Proof: Let $B=\left\langle b_{1}, \ldots, b_{n}\right\rangle$ and $C=\left\langle c_{1}, \ldots, c_{n}\right\rangle$ be isomorphic to $A$ such that $a_{i} \rightarrow b_{i}$ and $a_{i} \rightarrow c_{i}, i=1, \ldots, n$ induce isomorphisms and form the HNN extensions

$$
\begin{aligned}
& U=\left\langle t, A ; t^{-1} a_{i} \mu t=a_{i} \nu, i=1, \ldots, n\right\rangle, \\
& V=\left\langle s, B \times C ; s^{-1} b_{i} \mu s=b_{i} \nu, s^{-1} c_{i} \nu, i=1, \ldots, n\right\rangle .
\end{aligned}
$$


Since $\mu$ and $\nu$ are not onto, we can find $a_{i} \in A-A \mu$ and $a_{j} \in A-A \nu$. Then by Britton's Lemma (see [2], p.181), the element $\left[t^{-1} a_{i} t, a_{j}\right]$ is not trivial and $t$ and $\left[t^{-1} a_{i} t, a_{j}\right]$ generate a free subgroup of $U$. Similarly $\left[s^{-1} b_{i} s, b_{j}\right]$ is not trivial and $s$ and $\left[s^{-1} b_{i} s, b_{j}\right]$ generate a free subgroup of $V$.

We can therefore construct the amalgamation

$$
G=\left\langle U, V ; t=\left[s^{-1} b_{i} s, b_{j}\right], s=\left[t^{-1} a_{i} t, a_{j}\right]\right\rangle
$$

We claim that the map $\vartheta$ from $G$ to $G \times G$ given on the generators by

$$
\begin{aligned}
& \vartheta: t \rightarrow(t, t), \vartheta: a_{i} \rightarrow\left(a_{i}, a_{i}\right), \quad i=1, \ldots, n, \\
& \vartheta: s \rightarrow(s, s), \vartheta: b_{i} \rightarrow\left(b_{i}, b_{i}\right), \quad i=1, \ldots, n, \\
& \vartheta: c_{i} \rightarrow\left(b_{i}\left(c_{i} \mu\right), c_{i} \mu\right), \quad i=1, \ldots, n,
\end{aligned}
$$

is a homomorphism and onto.

To show that $\vartheta$ is a homomorphism, we have to prove that $\vartheta$ maps relations in $G$ to relations of $G \times G$. Since all elements except $c_{i}, i=1, \ldots, n$, are mapped to the corresponding diagonal elements of $G \times G$, it suffices to show that $s^{-1} c_{i} \mu s=c_{i} \nu$, $i=1, \ldots, n$, and $\left[b_{i}, c_{j}\right]=1$, for $i, j=1, \ldots, n$, are mapped to relations.

Note,

$$
\begin{aligned}
\left(s^{-1} c_{i} \mu s\right) \vartheta & =\left(s^{-1} b_{i} \mu\left(c_{i} \mu^{2}\right) s, s^{-1} c_{i} \mu^{2} s\right) \\
& =\left(s^{-1} b_{i} \mu s s^{-1}\left(c_{i} \mu^{2}\right) s, s^{-1} c_{i} \mu^{2} s\right)
\end{aligned}
$$

This can be simplified by the relations of $V$ :

$$
\left(s^{-1} b_{i} \mu s s^{-1}\left(c_{i} \mu^{2}\right) s, s^{-1} c_{i} \mu^{2} s\right)=\left(b_{i} \nu c_{i} \mu \nu, c_{i} \mu \nu\right)
$$

We now use the fact that $\mu \nu=\nu \mu$ to derive $\left(b_{i} \nu c_{i} \mu \nu, c_{i} \mu \nu\right)=\left(b_{i} \nu c_{i} \nu \mu, c_{i} \nu \mu\right)$. But $\left(b_{i} \nu c_{i} \nu \mu, c_{i} \nu \mu\right)=\left(c_{i} \nu\right) \vartheta .\left[b_{i}, c_{j}\right] \vartheta=(1,1)$ is true because $B \times C$ is abelian.

It remains now to show that $\vartheta$ is onto. We observe, $\left[s^{-1} c_{i} s, b_{j}\right] \vartheta=\left(\left[s^{-1} b_{i} c_{i} \mu s, b_{j}\right]\right.$, $\left.\left[s^{-1} c_{i} \mu s, b_{j}\right]\right)=\left(\left[s^{-1} b_{i} s c_{i} \nu, b_{j}\right],\left[c_{i} \nu, b_{j}\right]\right)=\left(\left[s^{-1} b_{i} s, b_{j}, 1\right]\right)$. From the relations of $G$ we derive $(t, 1) \in G \vartheta$. Because $G \vartheta$ contains $\left(a_{i}, a_{i}\right), i=1, \ldots, n$, it contains $\left(a_{i} \mu, a_{i} \mu\right)$ for all $i$. Also $\left(a_{i} \mu, a_{i} \mu\right)(t, 1)^{-1}\left(a_{i} \mu, a_{i} \mu\right)^{-1}(t, 1)=\left(a_{i} \mu\left(a_{i} \nu\right)^{-1}, 1\right)$, $i=1, \ldots, n$. Since the elements $a_{i} \mu\left(a_{i} \nu\right)^{-1}, i=1, \ldots, n$ generate $A,(A, 1)$ is in $G \vartheta$. From $s=\left[t^{-1} a_{i} t, a_{j}\right]$, it follows that also $(s, 1) \in G \vartheta$. The consideration above with $s, B$ and $C$ shows now that $(B, 1)$ and $(C, 1)$ are in $G \vartheta$. Together we get that $(G, 1) \in G \vartheta$. If we look at the values of $\vartheta$ on the generators we see that therefore $(1, t),(1, s),\left(1, a_{i}\right),\left(1, b_{i}\right),\left(1, c_{i} \mu\right), i=1, \ldots, n$, are in $G \vartheta$. As before we conclude in a similar way that $(1, G) \in G \vartheta$. This completes the proof of Theorem 3 . 
The next example is the group constructed by Baumslag and Miller [1].

EXAMPLE 4: Let $A=\langle a\rangle$ be infinite cyclic, and $\mu, \nu$ given by $a \mu=a^{2}, a \nu=a^{3}$. Then the conditions of the theorem are satisfied. Also $a \in A-A \mu$ and $a \in A-A \nu$. By Theorem 3 or rather the proof of Theorem 3, we construct the groups

$$
\begin{aligned}
& U=\left\langle t, a ; t^{-1} a^{2} t=a^{3}\right\rangle \\
& V=\left\langle s, b, c ;[b, c]=1, s^{-1} b^{2} s=b^{3}, s^{-1} c^{2} s=c^{3}\right\rangle
\end{aligned}
$$

and conclude that for the group

$$
\begin{array}{r}
G_{4}=\left\langle t, a, s, b, c ; t^{-1} a^{2} t=a^{3},[b, c]=1, s^{-1} b^{2} s=b^{3}, s^{-1} c^{2} s=c^{3},\right. \\
\left.s=\left[t^{-1} a t, a\right], t=\left[s^{-1} b s, b\right]\right\rangle
\end{array}
$$

the map $\vartheta$ from $G_{4}$ to $G_{4} \times G_{4}$ given by

$$
\begin{aligned}
& \vartheta: t \rightarrow(t, t), \vartheta: a \rightarrow(a, a), \vartheta: s \rightarrow(s, s), \vartheta: b \rightarrow(b, b), \\
& \vartheta: c \rightarrow\left(b c^{2}, c^{2}\right)
\end{aligned}
$$

is a homomorphism and onto.

EXAMPLE 5: Let $A=\left\langle a_{1}, a_{2}\right\rangle$ be free abelian on two generators, and $\mu, \nu$ given by $a_{1} \mu=a_{1}^{2}, a_{2} \mu=a_{2}, a_{1} \nu=a_{1}, a_{2} \nu=a_{2}^{2}$. Then the conditions of the theorem are satisfied. Also $a_{1} \in A-A \mu$ and $a_{2} \in A-A \nu$.

Again by the proof of Theorem 3 , we construct the groups

$$
\begin{gathered}
U=\left\langle t, a_{1}, a_{2} ;\left[a_{1}, a_{2}\right]=1, t^{-1} a_{1}^{2} t=a_{1}, t^{-1} a_{2} t=a_{2}^{2}\right\rangle, \\
V=\left\langle s, b_{1}, b_{2}, c_{1}, c_{2} ;\left[b_{1}, b_{2}\right]=1, s^{-1} b_{1}^{2} s=b_{1}, s^{-1} b_{2} s=b_{2}^{2},\right. \\
{\left[c_{1}, c_{2}\right]=1, s^{-1} c_{1}^{2} s=c_{1}, s^{-1} c_{2} s=c_{2}^{2},} \\
\left.\left[b_{i}, c_{j}\right]=1, i, j=1,2\right\rangle
\end{gathered}
$$

and conclude that for the group

$$
\begin{array}{r}
G_{5}=\left\langle t, a_{1}, a_{2}, s, b_{1}, b_{2}, c_{1}, c_{2} ;\left[a_{1}, a_{2}\right]=1, t^{-1} a_{1}^{2} t=a_{1}, t^{-1} a_{2} t=a^{2},\right. \\
{\left[b_{1}, b_{2}\right]=1, s^{-1} b_{1}^{2} s=b_{1}, s^{-1} b_{2} s=b_{2}^{2},} \\
{\left[c_{1}, c_{2}\right]=1, s^{-1} c_{1}^{2} s=c_{1}, s^{-1} c_{2} s=c_{2}^{2},} \\
{\left[b_{i}, c_{j}\right]=1, i, j=1,2,} \\
\left.s=\left[t^{-1} a_{1} t, a_{2}\right], t=\left[s^{-1} b_{1} s, b_{2}\right]\right\rangle
\end{array}
$$

there exists a homomorphism $\vartheta$ from $G_{5}$ onto $G_{5} \times G_{5}$. 


\section{Finitely generated groups $G \cong G \times G$}

THEOREM 4. If a non-trivial finitely generated group $G$ has a homomorphism $\vartheta$ from $G$ onto $G \times G$, then there exists a non-trivial quotient $E$ of $G$ isomorphic to its own direct square.

Proof: Let $K$ be a maximal normal subgroup, $K \neq G$, such that, $K \vartheta$ is contained in $K \times K$. Such a normal subgroup exists for a finitely generated group $G$ by Zorn's lemma. Set $E=G / K$. Then $\vartheta$ induces a map $\eta$ of $E$ onto $E \times E$. Let $L=\operatorname{ker} \eta$; then $L \neq E$ and its preimage $N$ under the quotient map $G \rightarrow E$ contains $K$ and has the property that $N \vartheta$ is contained in $N \times N$. By maximality of $K$, we get that $K=N$ and therefore that $\eta$ is an isomorphism. This completes the proof of Theorem 4.

As in Section 3 we can find a normal subgroup $K^{*}$ with $G / K^{*} \cong G / K^{*} \times G / K^{*}$ as follows. Let $K_{1}=\operatorname{ker}(\vartheta)$; then $\vartheta$ induces a map $\vartheta_{1}$ of $G / K_{1}$ onto $G / K_{1} \times G / K_{1}$. Let $K_{2}$ be the preimage of $\operatorname{ker}\left(\vartheta_{1}\right)$ under the quotient map $G$ to $G / K_{1}$. Inductively if $\vartheta_{i}$ maps $G / K_{i}$ onto $G / K_{i} \times G / K_{i}$ we define $K_{i+1}$ to be the preimage of $\operatorname{ker}\left(\vartheta_{i}\right)$ under the quotient map $G$ to $G / K_{i}$. The $K_{i}$ form an ascending chain of subgroups. Let $K^{*}=\cup K_{i}$. Then $\vartheta$ induces a map $\vartheta^{*}$ from $G / K^{*}$ onto $G / K^{*} \times G / K^{*}$ which is an isomorphism.

PROPOSITION 3. Let $\vartheta$ be a homomorphism of $G$ onto $G \times G$. If $N$ is a normal subgroup of $G$ such that $N \vartheta$ is contained in $N \times N$ and $\vartheta$ induces an isomorphism of $G / N$ to $G / N \times G / N$, then $N \supseteq K^{*}$ for $K^{*}=\cup K_{i}$ as above.

Proof: Obviously, $N \supseteq K_{1}=\operatorname{ker}(\vartheta)$. Assume now that $N \supseteq K_{i}$. Then $K_{i+1} N / N$ is in the kernel of the homomorphism $G / N$ to $G / N \times G / N$ induced by $\vartheta$. Since this map is an isomorphism, $N \supseteq K_{i+1}$. Therefore by induction, $N \supseteq K_{i}$ for all $i$ and hence $N \supseteq K^{*}$.

\section{REFERENCES}

[1] G. Baumslag and C.F. Miller III, 'Some odd finitely presented groups', Bull. London Math. Soc. 20 (1988), 239-244.

[2] R.C. Lyndon and P.E. Schupp, Combinatorial Group Theory (Springer-Verlag, Berlin, Heidelberg, New York, 1977).

[3] D. Meier, 'Non-hopfian groups', J. Lond Math. Soc. 26 (1982), 265-270.

[4] J.M. Tyrer Jones, 'Direct products and the Hopf property', J. Austral. Math. Soc. 17 (1974), 174-196. 